\title{
Head and neck radiotherapy amid the COVID-19 pandemic: practice recommendations of the Italian Association of Radiotherapy and Clinical Oncology (AIRO)
}

\author{
Daniela Alterio $^{1}$-Stefania Volpe ${ }^{1,2}$ (1) . Almalina Bacigalupo ${ }^{3} \cdot$ Pierluigi Bonomo $^{4} \cdot$ Francesca De Felice $^{5}$. \\ Francesco Dionisi ${ }^{6}$. Ida D'Onofrio ${ }^{7}$. Elisa D'Angelo ${ }^{8}$. Alessia Di Rito ${ }^{9}$. Giuseppe Fanetti ${ }^{10}$. Pierfrancesco Franco ${ }^{11}$. \\ Marta Maddalo ${ }^{12}$ - Anna Merlotti ${ }^{13}$. Francesco Micciché ${ }^{14}$. Ester Orlandi ${ }^{15}$. Fabiola Paiar ${ }^{16}$. Stefano Ursino ${ }^{17}$. \\ Matteo Pepa ${ }^{1} \cdot$ Renzo Corvò $^{3,18}$. Nadia Gisella Di Muzio ${ }^{19,20}$. Stefano Maria Magrini ${ }^{12}$. Elvio Russi ${ }^{13}$. \\ Giuseppe Sanguineti $^{21} \cdot$ Barbara Alicja Jereczek-Fossa $^{1,2} \cdot$ Vittorio Donato $^{22,23} \cdot$ Daniela Musio ${ }^{5,24}$
}

Received: 24 June 2020 / Accepted: 5 August 2020 / Published online: 17 August 2020

๑) Springer Science+Business Media, LLC, part of Springer Nature 2020

\begin{abstract}
Management of patients with head and neck cancers (HNCs) is challenging for the Radiation Oncologist, especially in the COVID-19 era. The Italian Society of Radiotherapy and Clinical Oncology (AIRO) identified the need of practice recommendations on logistic issues, treatment delivery and healthcare personnel's protection in a time of limited resources. A panel of 15 national experts on HNCs completed a modified Delphi process. A five-point Likert scale was used; the chosen cut-offs for strong agreement and agreement were $75 \%$ and $66 \%$, respectively. Items were organized into two sections: (1) general recommendations (10 items) and (2) special recommendations (45 items), detailing a set of procedures to be applied to all specific phases of the Radiation Oncology workflow. The distribution of facilities across the country was as follows: $47 \%$ Northern, 33\% Central and 20\% Southern regions. There was agreement or strong agreement across the majority (93\%) of proposed items including treatment strategies, use of personal protection devices, set-up modifications and follow-up re-scheduling. Guaranteeing treatment delivery for HNC patients is well-recognized in Radiation Oncology. Our recommendations provide a flexible tool for management both in the pandemic and post-pandemic phase of the COVID-19 outbreak.
\end{abstract}

Keywords COVID-19 pandemic $\cdot$ Head and neck cancers $\cdot$ Radiation oncology $\cdot$ Clinical practice recommendations

\section{Introduction}

Management of head and neck cancer (HNC) during the COVID-19 outbreak is challenging [1]. Not only patients diagnosed with HNC are often comorbid and frail [2-4], but may have specific features increasing their risk of being more severely affected by COVID-19 infection [5]. Treatment-related sequelae, including but not limited to

Vittorio Donato and Daniela Musio equally contributed to the current work and should be considered as co-last Authors.

Electronic supplementary material The online version of this article (https://doi.org/10.1007/s12032-020-01409-2) contains supplementary material, which is available to authorized users.

Stefania Volpe

stefania.volpe@ieo.it

Extended author information available on the last page of the article the presence of tracheostomy [5] and the myelosuppressive action of chemo- and radiotherapy (RT) [6], further increase the threat of the pandemic on these patients' health. RT represents a mainstay in $\mathrm{HNC}$, both in the definitive and in the post-operative setting [7]. While the unprecedented COVID19 emergency is requiring health care professionals to redefine treatment paradigms, there is a general agreement on three basic principles for HNC management in Radiation Oncology: (1) curative-intent RT should be considered as non-deferrable [8], (2) treatment breaks should be minimized to preserve patients' outcomes $[9,10]$, and (3) maintenance of adequate quality standards should be guaranteed, especially when highly conformal techniques are used [7,8].

COVID-19 binds to the epithelial cells in the nasal cavities and the oropharynx [11]. Therefore, as compared with other healthcare professionals, those managing HNC patients are at higher risk of being exposed to aerosolized droplets by asymptomatic carriers [12]. Therefore, if access 
to oncological care has to be guaranteed even during the most severe phases of the COVID-19 outbreak, personnel protection needs to be maximized and viral transmission prevented $[13,14]$. As for surgical treatments, patients undergoing RT may be required to remove their medical masks for either physical examination or set-up procedures (e.g. thermoplastic mask positioning), thus requiring additional cautions by healthcare providers. Since swabs-based screenings are hardly extendable to the whole population [15], it is advisable to consider all patients as COVID-19 asymptomatic carriers and to define adequate personal and environmental protective measurements.

Italy was the first country in Europe hit by the COVID19 pandemic [16]. Despite significant differences in contagion rates were found across the country, with Northern regions (namely, Lombardy, Piedmont, Emilia Romagna and Veneto) being the most severely affected areas, all Italian Radiation Oncology facilities were called to respond to the necessity of treating patients at the time of COVID-19 outbreak [17]. The Italian Association of Radiotherapy and Clinical Oncology (Associazione Italiana di Radioterapia ed Oncologia Clinica, AIRO) has produced a set of general indications and recommendations dedicated to Italian Radiation Oncologists, which are constantly updated and publicly available in Italian and English languages [18-20]. Additionally, dedicated HNC practice recommendations have been recently published thanks to the joint efforts of the American and European Societies of Radiation Oncology (ASTRO and ESTRO, respectively) to optimize indication to treatment of COVID-19 positive HNC patients [8].

However, recommendations for the re-organization of Radiation Oncology Departments managing HNC are still lacking. With countries such as the USA, Brazil and the Russian Federation still being amid the pandemic peak as of June 22th, there is a persistent need of defining effective and standardized management policies for this subset of patients. Therefore, based on single institutional experiences and on currently available guidelines [8,21-23], AIRO decided to call national HNC experts to a consensus on practical management of HNC patients facing the COVID-19 pandemic. Aim of the current work was to foster discussion among Italian Fellow Radiation Oncologists, to promote high-quality strategies for $\mathrm{HNC}$ and to provide facilities both in the pandemic and in the post-pandemic phase with a practical tool for daily clinical practice.

\section{Methods}

Under endorsement of the AIRO Executive Committee, a panel of 15 Radiation Oncologists with well-recognized expertise in HNC, drafted a preliminary set of items focusing on patients' management and healthcare personnel's protection at the time of the COVID-19 pandemic. Overall, recommendations were tailored on patients without any COVID-19 related symptoms; all patients were therefore considered as potential carriers requiring specific cautions to minimize the risk of cross-infection between patients and health care providers. The whole RT workflow was considered, from first outpatient consultation to follow-up procedures; special attention was given to the sanitization of personal protective equipment (PPE) and sanitization measures of the work environment.

Conversely, Institutional and/or Departmental procedures applied for all oncologic patients (i.e. phone/clinical triage, temperature measurement at the entrance of the hospital) are not discussed in the present consensus as well as in patients' management. Due to time constraints related to the quickly evolving pandemic scenario, a systematic literature review was not performed. Nevertheless, available evidence was considered [8, 12, 21, 22].

Items were organized into two sections: (1) general recommendations and (2) special recommendations, detailing a set of procedures to be applied to all specific phases of the RT workflow.

On May 10th, 15 Radiation Oncologists with recognized expertise in HNC RT from the major Italian facilities were invited to participate in this project. By May 15th, they had all confirmed their availability. A modified Delphi process was used to grade consensus about each proposed item [24]. A five-point Likert scale was chosen to grade agreement or disagreement, with possibilities of vote encompassing the following: "strongly agree", "agree", "neutral", "disagree" and "strongly disagree". All votes were given in anonymous form.

The facilitator (DA) circulated the first consensus draft among the participants; modification of original items and/ or inclusion of additional topics was allowed in a preliminary round, to meet participants' comments and suggestions. Once items were defined, they were circulated using the online survey tool Google Forms [25]. Responders had $36 \mathrm{~h}$ to complete the first round and $24 \mathrm{~h}$ for the second one. In case an item reached the agreement on the first round, it was withdrawn from the second. Conversely, in case consensus failed to reach a $66 \%$ threshold, it could be edited according to the panel suggestions, and be re-circulated in the second round.

A cut-off of $75 \%$ was set to define "strong agreement", while "agreement" was considered for items reaching at least $66 \%$ of consensus among participants [24].

The final consensus was submitted to 5 senior supervisors (RC, NGDM, SMM, ER, GS), and approved by the AIRO Scientific Committee and Board. 


\section{Results}

All invited Radiation Oncologists accepted to participate to the consensus, which reached a $100 \%$ response rate in both rounds. The majority of participants $(60 \%)$ was aged between 40 and 50 years and was equally distributed among genders, with a slight female predominance $(60 \%$ vs $40 \%)$. Seventy-three percent had more than 10 years of experience in HNC management. The distribution of RT facilities across the country was as follows: $47 \%$ Northern, 33\% Central and 20\% Southern Italian regions. Sixtyseven percent of responders were affiliated to University or Research Hospitals, while private and single-specialty centers represented the $13 \%$ of the sample. Twenty-seven percent of centers were public hospitals.
The number of items was 55 and 4 in respective rounds. Panelists' responses to each question are detailed in Supplementary Materials.

\section{General recommendations}

Ten items on General Recommendations were structured as following: (1) HNC patients, (2) healthcare professionals, (3) Radiation Oncology Department organization and (4) treatment delivery strategies. All but two items (87\%) reached either a strong agreement or agreement during the first round. The two rejected items were re-circulated during the second round, and both failed to achieve a sufficient level of agreement among panelists. Items with the resulting strength of agreement are listed in Table 1.

Table 1 Strength of Agreement for General Recommendations in HNC Radiation Oncology in the COVID-19 pandemic

\begin{tabular}{|c|c|c|c|}
\hline Question & Strength of agreement & $\%$ of agreement ${ }^{\mathrm{a}}$ & Median $^{\mathrm{b}}$ \\
\hline \multicolumn{4}{|l|}{ 1. Head and neck cancer patients } \\
\hline $\begin{array}{l}\text { 1.1. Patients should be required to wear a medical mask to cover their nose and mouth; } \\
\text { the medical mask should be maintained during any phase of patient's permanence at the } \\
\text { Radiation Oncology Department unless otherwise specified by the healthcare personnel }\end{array}$ & Strong agreement & 100 & 1 \\
\hline $\begin{array}{l}\text { 1.2 A second medical mask should be worn in case the patient has a tracheostomy; the } \\
\text { second medical mask should be maintained during any phase of patient's permanence at } \\
\text { the Radiation Oncology Department unless otherwise specified by the healthcare person- } \\
\text { nel }\end{array}$ & Strong agreement & 93.3 & 1 \\
\hline $\begin{array}{l}\text { 1.3 Hand sanitization (by either direct hand sanitization with hydroalcoholic-based } \\
\text { disinfectants or by the use of gloves) should be performed, and repeated before each } \\
\text { procedure }\end{array}$ & Strong agreement & 100 & 1 \\
\hline $\begin{array}{l}\text { 1.4 Any swallowing and/or respiratory impairment which could exacerbate cough and } \\
\text { mucous secretion should be minimized whenever possible }\end{array}$ & Strong agreement & 93.3 & 1 \\
\hline $\begin{array}{l}\text { 1.5 Any swallowing and/or respiratory impairment which could increase the risk of ab- } \\
\text { ingestis pneumonitis should be minimized whenever possible }\end{array}$ & Strong agreement & 93.3 & 1 \\
\hline \multicolumn{4}{|l|}{ 2. Health care professionals } \\
\hline $\begin{array}{l}\text { 2.1 Any medical and technical procedures requiring the patient to remove his/her medical } \\
\text { mask should be considered at high risk of viral dissemination }\end{array}$ & Strong agreement & 86.7 & 1 \\
\hline $\begin{array}{l}\text { 2.2 Health care professionals (Medical Doctors, Radiation Therapists and Nurses) involved } \\
\text { in the management of HNC patients should wear gloves, goggles (or, alternatively, a face } \\
\text { shield), gowns, respirators (i.e., N95 or FFP2 standard or equivalent) and aprons in any } \\
\text { phase of the RT course }\end{array}$ & Agreement & 66.7 & Equal \\
\hline \multicolumn{4}{|l|}{ 3. Radiation oncology department organization } \\
\hline $\begin{array}{l}\text { 3.1 At least one consultation room should be dedicated to weekly HNC patients examina- } \\
\text { tions during RT delivery for radiation-related acute toxicities (in case medical mask } \\
\text { removal is required) }\end{array}$ & No agreement & 60 & - \\
\hline $\begin{array}{l}\text { 3.2 At least one consultation room should be dedicated for } \mathrm{HNC} \text { patients nursing care (i.e. } \\
\text { skin medications, in case medical mask removal is required) }\end{array}$ & Agreement & 73.3 & 2 \\
\hline $\begin{array}{l}\text { 3.3 Extra sanitization procedures according to Institutional policies should be applied for } \\
\text { rooms dedicated to HNC patients } s^{c}\end{array}$ & No agreement & 60 & - \\
\hline
\end{tabular}

$F F P 2$ class II filtering facepiece, $H N C$ head and neck cancer, $R T$ radiotherapy

a\% of agreement is computed as the sum of responses "strongly agree" and "agree"

bMedian can be either 1, if "strongly agree" answers prevailed, 2, if "agree" answers prevailed or "equal" if neither prevailed

${ }^{\mathrm{c}}$ These questions were proposed again in the second round but agreement was not reached 


\section{Special recommendations}

Forty-five items were categorized as special recommendations, and were organized as following: (1) indication to treatment, (2) first outpatient consultation, (3) computed tomography (CT) simulation procedure, (4) treatment delivery strategy, (5) RT treatment sessions (6) in-treatment consultations, (7) patients with suspected COVID-19 infection, and (8) follow-up consultations.

Of these, $36 / 40(90 \%)$ reached the consensus at the end of the first round. Two items were re-circulated during the second round: one was slightly edited following the panel's requirement (item 5.3, "Indication to treatment": an example of alternative treatment strategy for early-stage HNC was provided), and was approved during the second round with a strong level of agreement. Another item (6.5, "First Outpatient Consultation") reached the agreement during the second round.

Two items were withdrawn from the second round by the facilitator to meet panelists' observations. Specifically, one item, included among the "Computer tomography (CT) simulation procedures", considered the possibility of positioning the medical masks over the thermoplastic mask in case it was not possible for the patient to wear it under the immobilization device. More than one quarter of the responders disagreed with the statement, while one third rated it as "neutral". As no suggestions nor comments were provided for item modification, it was not admitted to the second round. The second excluded statement (originally included among the "Patients with suspected COVID-19 infection" sub-section) was formulated as following: "In case of treatment interruption for COVID-19 infection, at least two negative nasal and oropharyngeal swab tests are required before the patient can be re-admitted to the hospital". Despite the statement mirrored local health policies, it was judged both as hardly generalizable and not in line with existing recommendations [8], and was therefore removed after the first round.

Items at the end of the two rounds are collected in Table 2 with the corresponding level of agreement.

\section{Discussion}

The aim of these practice recommendations by the AIRO was to provide a decisional framework for Radiation Oncologists treating HNC patients at the time of the COVID-19 pandemic. While in Italy the lockdown is progressively being mitigated for the general population, hospitals remain at high risk of cross-contamination for patients and healthcare professionals [12, 26, 27]. With a number of COVID19-positive cases in Italy of 230.000 cases as of May 25th
2020 and a potentially higher number of undiagnosed asymptomatic carriers, it is critical to maintain the highest quality of safety procedures when treating HNC malignancies. With this awareness, we propose this set of recommendations for every-day clinical workflow.

Relevant phases of Radiation Oncology workflow were reviewed, discussed and optimized in the light of the Italian experience of the last 3 months.

Given the different distribution of COVID-19 incidence and prevalence across the country, the panel was required to evaluate the issue of patients' relocation in another region for RT delivery. While responders generally agreed on the possibility of recommending treating facilities close to the patient's domicile, a strong degree of consensus was not reached. Specifically, responders suggested considering high-volume centers whenever possible, as both RT technique and treating Radiation Oncologist's experience have been associated with better oncological outcomes [28].

Overall, the panel underlined that multidisciplinary team meetings should be maintained to discuss individual indication to RT. As the issue of locally-advanced disease had already been analyzed [29], it was not proposed to the current panel. Conversely, the possibility of offering alternative treatment strategies was discussed and approved for early-stage tumors, such as mini-invasive surgery for T1-T2 cancers of the glottis. Indeed, although hypofractionated schemes may be proposed, 20 is the lowest currently accepted number of fractions for curative-intent treatments [8]. Therefore, the choice to address to mini-invasive surgery would have the advantage to prevent patients from multiple accesses to the hospital and therefore limit the risk of crosscontamination. Considering the palliative setting [8], the prescription of extreme hypofractionation (even in a single fraction of $8 \mathrm{~Gy}$ ) could optimize the ratio between the risk of infection and the expected benefits of irradiation.

Agreement was found on patients' clinical management from the first outpatient consultation by the Radiation Oncologist to follow-up scheduling. Some observations by the responders focused on the presence of accompanying persons during the RT workflow. Respecting social distancing measures was recognized as a priority in all environments. To this aim, caregivers' access to the Radiation Oncology Department should be discouraged. However, the panel recognized that a caregiver could attend medical consultations in selected cases (i.e. senior individuals, speech impairment, and linguistic barriers). As the reduction of psychological distress has been correlated with increased compliance to treatment and better oncological outcomes [30], caution in balancing strict safety policies and support from the caregivers is advised for this subset of patients.

While Italian practical recommendations on general reorganization policies of Radiation Oncology Departments have been already provided [20], we focused on additional 
Table 2 Strength of agreement for special recommendations in HNC Radiation Oncology in the COVID-19 pandemic

5. Indication to treatment

5.1 Multidisciplinary team meetings should be maintained in compliance of social distanc- Strong agreement ing rules or via tele-meetings

5.2 Treatment indications should comply with the recently published ASTRO/ESTRO practice recommendations

5.3 Alternative treatment strategies should be considered for early-stage $\mathrm{HN}$ tumors (i.e. $\quad$ Strong agreement ${ }^{\mathrm{c}}$ endoscopic surgery for early glottis tumors)

5.4 Indication to palliative treatments should be weighed against the increased risk of viral Strong agreement exposure in the hospital environment

\author{
Strong agreement
}

100

1

6. First outpatient consultation

6.1 A phone triage should be performed before patients are admitted in the Hospital

6.2 Clinical history on COVID-19 related symptoms should be collected

6.3 Close contact with COVID-19 positive cases should be collected

6.4 In case of suspected COVID-19 infection, patients should be tested (with nasal/oropharyngeal swabs preceding clinical examination)

6.5 Patients who are not resident in the same region as the treating facility should be invited to seek for a Radiation Oncology consultation and to perform RT close to their domicile

6.6 At least medical beds and room equipment should be cleaned with hydroalcoholic disinfectants after each use

6.7 Extra sanitization procedures according to Institutional policies should be applied for examination rooms dedicated to $\mathrm{HNC}$ patients

6.8 During the clinical examination, any accompanying person should be invited to wait outside the Department

7. CT simulation procedures

7.1 Clinical history on COVID-19 related symptoms should be collected before the CT simulation procedure

7.2 Close interaction with COVID-19 positive contacts should be collected before the CT simulation procedure

7.3 In case patients need to remove his/her medical mask, health care professionals should be considered at high risk for COVID-19 infection and equipped accordingly

7.4 Thermoplastic masks as well as all set-up devices should be cleaned with hydroalcoholic-based disinfectants after each procedure

7.5 Set-up devices should be used according to the RT technique of choice, and according to standard Departmental procedures

7.6 If patients need to remove his/her medical mask, the simulation CT room should be cleaned in compliance with a structured sanitation protocol

7.7 CT simulation scheduling should be arranged to allow adequate room sanitization according to Institutional policies

7.8 Should it be unfeasible for the patient to wear his/her medical mask under the thermoplastic mask, an attempt should be made to position the surgical mask above the thermoplastic mask

7.9 After each procedure, health care professionals have to remove gloves, wash their hands accurately, sanitize their goggles (or face shields) with hydroalcoholic solution

7.10 All thermoplastic masks and individual set-up devices should be stored in protective disposable bags

8. Treatment delivery strategy

8.1 Treatment planning strategies (i.e. treatment technique) should be maintained according to Departmental standard of care

8.2 In case any modification of set-up is required (i.e. cutting the thermoplastic mask to improve patients' tolerance to RT, if needed), the possibility of higher uncertainties in patient's positioning should be considered and managed as needed

8.3 In case of any modification of the thermoplastic mask, it is advised to maintain a strong fixation of at least the patient's chin and nose

\begin{tabular}{|c|c|c|}
\hline Strong agreement & 86.7 & 1 \\
\hline Strong agreement & 93.3 & 1 \\
\hline Strong agreement & 93.3 & 1 \\
\hline Strong agreement & 93.3 & 1 \\
\hline Agreement $^{\mathrm{d}}$ & 66.7 & Equal \\
\hline Strong agreement & 86.7 & 1 \\
\hline Agreement & 66.7 & 2 \\
\hline Strong agreement & 86.7 & 2 \\
\hline Strong agreement & 86.7 & 1 \\
\hline Strong agreement & 80 & 1 \\
\hline Strong agreement & 80 & 2 \\
\hline Strong agreement & 93.3 & 1 \\
\hline Strong agreement & 100 & 1 \\
\hline Strong agreement & 80 & 1 \\
\hline Strong agreement & 80 & 2 \\
\hline No agreement & 40 & - \\
\hline Strong agreement & 93.3 & 1 \\
\hline Strong agreement & 80 & 1 \\
\hline Strong agreement & 100 & 1 \\
\hline Strong agreement & 100 & 1 \\
\hline Strong agreement & 100 & 1 \\
\hline
\end{tabular}


Table 2 (continued)

Question Strength of agreement $\%$ of agreement ${ }^{\mathrm{a}}$ Median $^{\mathrm{b}}$

8.4 In case any significant modification of set-up devices (i.e. omission of tools such as the Strong agreement mouth-piece bite), the subsequent CTV-PTV margins should be modified to account for set-up uncertainties

8.5 In case of any modification of the thermoplastic mask it is recommended to applied more strict set-up protocols (i.e. daily image guided radiotherapy)

9. RT treatment sessions

9.1 Patients should not be accompanied in the Radiation Oncology waiting room, unless strictly necessary (i.e. language barriers)

9.2 In case several patients are in the same waiting room, adequate social distancing procedures should be respected

9.3 In case patients need to remove his/her medical mask, health care professionals should be considered at high risk for COVID-19 infection and equipped accordingly

9.4 Thermoplastic masks as well as all set-up devices should be cleaned with hydro alcoholic-based disinfectants after each procedure

9.5 All thermoplastic masks and individual set-up devices should be stored in protective disposable bags

10. In-treatment consultations

10.1 Acute RT-related toxicity should be treated according to the Departmental standard of care

10.2 Patients should be examined at least once per week for RT-related acute toxicity assessment

10.3 Clinical history on COVID-19 related symptoms should be collected during each consultation

10.4 Close interaction with COVID-19 positive contacts should be collected during each consultation

10.5 Patients should be trained to perform basic skin medications on their own to minimize medical mask removal during the course of treatment

10.6 Both the consultation room(s) and the Infirmary should be sanitized according to Institutional policies

11. Suspected COVID-19 infection

11.1 In case of suspected COVID-19 infection, patients should be tested by nasal and oropharyngeal swab test

11.2 In case of COVID-19 infection, clinicians are invited to comply with ASTRO/ ESTRO practice recommendations

11.3 In case of treatment interruption for COVID-19 infection, at least two negative nasal and oropharyngeal swab tests are required before the patient can be re-admitted to the Hospital

11.4 In case of prolonged treatment interruptions for severe COVID-19 infection, a multidisciplinary discussion should either confirm or re-define the patient's program

12. Follow-up consultations

12.1 For patients at low risk of loco-regional recurrence and without relevant symptoms, telehealth surveillance should be performed via phone calls to assess clinical status and results of prescribed radiological examinations

12.2 Face-to-Face consultations should be maintained in case of either (1) need for clinical Strong agreement evaluation of tumor response to treatment, (2) high-risk of local recurrence, (3) patients with reporting any cancer-related symptoms and/or signs

12.3 During the follow up consultations, the above-mentioned recommendations for the first outpatient consultations should be respected

ASTRO American Society for Radiation Oncology, CT computed tomography, CTV clinical target volume, ESTRO European Society for Radiotherapy and Oncology, $H N$ head and neck, $H N C$ HN cancer, $P T V$ planning target volume, $R T$ radiotherapy

a\% of agreement is computed as the sum of responses "strongly agree" and "agree"

bMedian can be either 1, if "strongly agree" answers prevailed, 2, if "agree" answers prevailed or "equal" if neither prevailed

${ }^{\mathrm{c}}$ Modified between 1st and 2nd round to address some comments from responders. Strong agreement reached after 2 nd round

${ }^{\mathrm{d}}$ Agreement was reached after 2 nd round 
issues specific to HNC care. Continuity of care was considered as a priority, with all experts recommending maintaining at least a weekly clinical examination for the assessment of RT-related acute toxicity. The panel recognized the importance of patients' compliance in observing general protection measurements [12]. Agreement, although with lesser strength (66.7\%), was reached on healthcare professionals protections. This might be due to different local policies on the use of PPE [17].

Allocation of consultation rooms was also debated: while the panel strongly agreed on reserving a room for nursing care (i.e. skin medications, in case medical masks removal is required), the allocation of a dedicated consultation room was not considered as relevant. Moreover, some panelist highlighted that nursing care of minor events (i.e. low-grade skin toxicity and mucositis) could be maintained in case expertise in $\mathrm{HNC}$ is already available.

General sanitization measurements have been diffusely implemented in Radiation Oncology facilities to decrease COVID-19 cross-infection [31]. Of note, HNC patients require a more frequent removal of their medical mask than other patients: the need of performing clinical examinations (i.e. oral cavity inspection, fibroscopy) exposes to a higher risk of environmental and personnel's contamination, together with the presence of cough and/or mucous secretions. As a consequence, the panel underlines the need of accurate disinfection of surfaces (i.e. room equipment, medical instrumentation) and RT set-up devices. Conversely, an agreement was not reached for extra-sanitization procedures (i.e. sanitization of medical rooms dedicated to $\mathrm{HNC}$ patients at the each working day). Admittedly, there is no current evidence that such procedures might be effective in further reducing the risk of cross-contamination when treating patients without COVID-19 relatable symptoms.

The panel showed a high consensus agreement when addressing items on set-up modifications: even in the midst of the COVID-19 emergency, priority should be maintained in avoiding any uncertainty in either treatment planning or delivery. Therefore, in case of modifications in immobilization devices (i.e. cutting of the thermoplastic mask), dedicated measurements should be applied, especially when high-conformal techniques with steep dose gradients are used [32, 33].

While the panel jointly considered first Radiation Oncology consultations as non-deferrable, distinctions are to be considered follow-up examinations. As intensive followup has not been associated with significant improvements in HNC patients' oncological outcomes [34], most of the responders agreed on re-scheduling follow-up consultations in those who are asymptomatic and/or at low-risk of local recurrence. Conversely, our recommendation is to maintain scheduled appointments in all other clinical scenarios, and whenever a direct assessment of clinical response to curative-intent RT is needed.

The recently published ASTRO/ESTRO practice guidelines [8] recommend to prioritize RT delivery in swabproven COVID-19 positive patients in case of mild symptomatic presentation who had already completed the first two weeks of treatment. Generally, the AIRO panelists agreed to comply with the above-described recommendations. However, no agreement was reached on the need of having two consecutive negative swab tests for re-admittance to RT. Heterogeneity was also found in indications to re-start RT among different centers who participated to the ASTRO/ESTRO consensus. Therefore, given the rarity of HNCs [35] and the above-detailed peculiarities of its management, the panel advices to perform systematic swabs test upon first patients' admittance in Radiation Oncology Department. This would allow to anticipate the diagnosis of COVID-19 positive cases to consider tailored treatment strategies.

Although we strongly believe in the value of consensusbased practice recommendations, some limitations should be considered. Firstly, if resources and working organization are not homogenous among treatment facilities, discrepancies among centers may be intensified in times of limited resources. Overall, it is not possible to make reliable predictions on the outbreak duration and severity, regardless of the pandemic phase. Consequently, clinicians may be forced to disregard our practice recommendations, despite the strong level of agreement we could reach for most of them. Flexibility and physicians' judgment should therefore be the guidance in finding the optimal balance between available resources and the best clinical practice at the safest conditions for healthcare providers.

Since Italy has been one the first and most severely affected countries, we hope that our hands-on consensus could be of value for other Fellow Colleagues still facing the COVID-19 outbreak while this manuscript is being written. As the epidemiological scenario is gradually improving in our country, novel regulations are currently under evaluation to adapt individual and institutional behaviors. We believe that our recommendations could also provide an easily adaptable tool for the upcoming post-pandemic phase, and meet the evolving needs of Radiation Oncologists treating HNC patients.

\section{Conclusion}

Based on the joint experience of the Italian Head and Neck Radiation Oncology community, this work provides a comprehensive set of clinical practice recommendations to be 
implemented in every phase of the RT workflow during the COVID-19 pandemic.

Acknowledgements This study was partially supported by the Italian Ministry of Health with "Progetto di Eccellenza". Stefania Volpe MD is a $\mathrm{PhD}$ student within the European School of Molecular Medicine (SEMM). The Authors thank the Scientific Committee and Board of the AIRO for the critical revision and final approval of the paper.

\section{Compliance with ethical standards}

Conflict of interest All authors declare that they have no conflict of interest.

\section{References}

1. De Felice F, Polimeni A, Tombolini V. The impact of Coronavirus (COVID-19) on head and neck cancer patients' care. Radiother Oncol. 2020;147:84-5. https://doi.org/10.1016/j.radon c.2020.03.020.

2. Yan F, Nguyen SA. Head and neck cancer: a high-risk population for COVID-19. Head Neck. 2020. https://doi.org/10.1002/ hed.26209.

3. Stordeur S, Schillemans V, Savoye I, et al. Comorbidity in head and neck cancer: is it associated with therapeutic delay, post-treatment mortality and survival in a population-based study? Oral Oncol. 2020;102:104561. https://doi.org/10.1016/j.oraloncolo gy.2019.104561.

4. Sharma A, Crosby DL. Special considerations for elderly patients with head and neck cancer during the COVID-19 pandemic. Head Neck. 2020. https://doi.org/10.1002/hed.26216.

5. Chow VLY, Chan JYW, Ho VWY, et al. Tracheostomy during COVID-19 pandemic_novel approach. Head Neck. 2020. https ://doi.org/10.1002/hed.26234.

6. The Head, and Neck Disease Site Group, Winquist E, Agbassi C, Meyers BM, Yoo J, Chan KKW. Systemic therapy in the curative treatment of head and neck squamous cell cancer: a systematic review. J Otolaryngol Head Neck Surg. 2017;46(1):29. https:// doi.org/10.1186/s40463-017-0199-x.

7. National Comprehensive Cancer Network. NCCN clinical practice guidelines in oncology—head and neck cancers. Version 1.2020. https://www.nccn.org/professionals/physician_gls/pdf/head-andneck.pdf. Accessed 22 June 2020.

8. Thomson DJ, Palma D, Guckenberger M, et al. Practice recommendations for risk-adapted head and neck cancer radiotherapy during the COVID-19 pandemic: an ASTRO-ESTRO consensus statement. Int J Radiat Oncol. 2020. https://doi.org/10.1016/j.ijrob p.2020.04.016.

9. Levy DA, Li H, Sterba KR, et al. Development and validation of nomograms for predicting delayed postoperative radiotherapy initiation in head and neck squamous cell carcinoma. JAMA Otolaryngol Neck Surg. 2020. https://doi.org/10.1001/jamao to.2020.0222.

10. González Ferreira JA, Jaén Olasolo J, Azinovic I, Jeremic B. Effect of radiotherapy delay in overall treatment time on local control and survival in head and neck cancer: review of the literature. Rep Pract Oncol Radiother. 2015;20(5):328-39. https://doi. org/10.1016/j.rpor.2015.05.010.

11. HCA Lung Biological Network, Sungnak W, Huang N, et al. SARS-CoV-2 entry factors are highly expressed in nasal epithelial cells together with innate immune genes. Nat Med. 2020. https:// doi.org/10.1038/s41591-020-0868-6.

12. Balakrishnan K, Schechtman S, Hogikyan ND, Teoh AYB, McGrath B, Brenner MJ. COVID-19 pandemic: what every otolaryngologist-head and neck surgeon needs to know for safe airway management. Otolaryngol Neck Surg. 2020. https://doi. org/10.1177/0194599820919751.

13. Ran L, Chen X, Wang Y, Wu W, Zhang L, Tan X. Risk factors of healthcare workers with Corona virus disease 2019: a retrospective cohort study in a designated hospital of Wuhan in China. Clin Infect Dis. 2020. https://doi.org/10.1093/cid/ciaa287.

14. Shuman AG, Campbell BH, AHNS Ethics \& Professionalism Service. Ethical framework for head and neck cancer care impacted by COVID-19. Head Neck. 2020. https://doi.org/10.1002/ hed.26193.

15. Rodriguez-Morales AJ, Cardona-Ospina JA, Gutiérrez-Ocampo E, et al. Clinical, laboratory and imaging features of COVID-19: a systematic review and meta-analysis. Travel Med Infect Dis. 2020;34:101623. https://doi.org/10.1016/j.tmaid.2020.101623.

16. Rosenbaum L. Facing Covid-19 in Italy-ethics, logistics, and therapeutics on the epidemic's front line. N Engl J Med. 2020;382(20):1873-5. https://doi.org/10.1056/NEJMp2005492.

17. Jereczek-Fossa BA, Palazzi MF, Soatti CP, et al. COVID-19 outbreak and cancer radiotherapy disruption in Lombardy, Northern Italy. Clin Oncol. 2020. https://doi.org/10.1016/j. clon.2020.04.007.

18. Associazione Italiana di Radioterapia Oncologica. Guidance document for risk assessment and management of patients and healthcare professionals in radiation oncology departments during ongoing COVID-19 spread. https://www.radioterapiaitalia.it/ wp-content/uploads/2020/04/LATEST_ENG_AIRO-COVID-19. pdf. Accessed 20 May 2020

19. Vavassori A, Tagliaferri L, Vicenzi L, et al. Practical indications for management of patients candidate to interventional and intraoperative radiotherapy (Brachytherapy, IORT) during COVID-19 pandemic - a document endorsed by AIRO (Italian Association of Radiotherapy and Clinical Oncology) Interventional Radiotherapy Working Group. Radiother Oncol. 2020;149:73-7. https ://doi.org/10.1016/j.radonc.2020.04.040.

20. Filippi AR, Russi E, Magrini SM, Corvò R. Letter from Italy: first practical indications for radiation therapy departments during COVID-19 outbreak. Int J Radiat Oncol. 2020. https://doi. org/10.1016/j.ijrobp.2020.03.007.

21. Fakhry N, Schultz P, Morinière $S$, et al. French consensus on management of head and neck cancer surgery during COVID-19 pandemic. Eur Ann Otorhinolaryngol Head Neck Dis. 2020. https ://doi.org/10.1016/j.anorl.2020.04.008.

22 Givi B, Schiff BA, Chinn SB, et al. Safety recommendations for evaluation and surgery of the head and neck during the COVID-19 pandemic. JAMA Otolaryngol Head Neck Surg. 2020. https://doi. org/10.1001/jamaoto.2020.0780.

23 Kowalski LP, Sanabria A, Ridge JA, et al. COVID-19 pandemic: effects and evidence-based recommendations for otolaryngology and head and neck surgery practice. Head Neck. 2020. https://doi. org/10.1002/hed.26164.

24. Hsu CC, Sandford BA. The Delphi technique: making sense of consensus. 2007. https://scholarworks.umass.edu/pare/vol12/iss1/. Accessed 22 June 2020.

25. Daniela A. COVID-19 and radiotherapy for head and neck cancer-an AIRO-endorsed consensus study. https://docs.googl e.com/forms/d/1xx5dhu-aoEYhjvNJe76G55al80gizSRz-0yowI 24J7c/edit. Accessed 22 June 2020.

26. Li R, Pei S, Chen B, et al. Substantial undocumented infection facilitates the rapid dissemination of novel coronavirus (SARS-CoV-2). Science. 2020;368(6490):489-93. https://doi. org/10.1126/science.abb3221. 
27 Yu J, Ouyang W, Chua MLK, Xie C. SARS-CoV-2 transmission in patients with cancer at a tertiary care hospital in Wuhan, China. JAMA Oncol. 2020. https://doi.org/10.1001/jamao ncol.2020.0980.

28. Naghavi AO, Echevarria MI, Strom TJ, et al. Patient choice for high-volume center radiation impacts head and neck cancer outcome. Cancer Med. 2018;7(10):4964-79. https://doi.org/10.1002/ cam4.1756.

29 Bhattacharjee A, Patil VM, Dikshit R, Prabhash K, Singh A, Chaturvedi P. Should we wait or not? The preferable option for patients with stage IV oral cancer in COVID-19 pandemic. Head Neck. 2020. https://doi.org/10.1002/hed.26196.

30. Chen AM, Hsu S, Felix C, Garst J, Yoshizaki T. Effect of psychosocial distress on outcome for head and neck cancer patients undergoing radiation: psychosocial distress and radiation therapy. The Laryngoscope. 2018;128(3):641-5. https://doi.org/10.1002/ lary.26751.

31. World Health Organization. Coronavirus disease (COVID-19) technical guidance: infection prevention and control/WASH. https ://www.who.int/emergencies/diseases/novel-coronavirus-2019/ technical-guidance/infection-prevention-and-control. Accessed 22 June 2020.

32. Yanagihara Ted K, Holland Roger E, Chera Bhisham S. Practical challenges of mask-to-mask encounters with patients with head

\section{Affiliations}

\section{Daniela Alterio $^{1}$-Stefania Volpe ${ }^{1,2}$ (1) $\cdot$ Almalina Bacigalupo $^{3} \cdot$ Pierluigi Bonomo $^{4} \cdot$ Francesca De Felice $^{5}$. Francesco Dionisi ${ }^{6}$. Ida D'Onofrio ${ }^{7}$. Elisa D'Angelo ${ }^{8}$. Alessia Di Rito ${ }^{9}$. Giuseppe Fanetti ${ }^{10}$. Pierfrancesco Franco ${ }^{11}$. Marta Maddalo ${ }^{12}$. Anna Merlotti ${ }^{13}$. Francesco Micciché ${ }^{14}$. Ester Orlandi ${ }^{15}$. Fabiola Paiar ${ }^{16}$. Stefano Ursino ${ }^{17}$. Matteo Pepa ${ }^{1} \cdot$ Renzo Corvò ${ }^{3,18} \cdot$ Nadia Gisella Di Muzio ${ }^{19,20}$. Stefano Maria Magrini ${ }^{12}$. Elvio Russi ${ }^{13}$. Giuseppe Sanguineti ${ }^{21} \cdot$ Barbara Alicja Jereczek-Fossa ${ }^{1,2} \cdot$ Vittorio Donato $^{22,23} \cdot$ Daniela Musio $^{5,24}$}

1 Division of Radiation Oncology, IEO, European Institute of Oncology IRCCS, Milan, Italy

2 Department of Oncology and Hemato-Oncology, University of Milan, Milan, Italy

3 Radiation Oncology Policlinico San Martino IRCCS, Genova, Italy

4 Radiation Oncology, Azienda Ospedaliero-Universitaria Careggi, Florence, Italy

5 Department of Radiotherapy, Policlinico Umberto I, "Sapienza" University of Rome, Rome, Italy

6 Proton Therapy Unit, Department of Oncology, Azienda Provinciale Per I Servizi Sanitari, APSS, 38123 Trento, Italy

7 Unit of Radiation Oncology, Ospedale del Mare, Naples, Italy

8 Radiation Oncology Department, University Hospital of Modena, Modena, Italy

9 Radiotherapy Unit, Ospedale "Mons. A.R. Dimiccoli”, Bari, Italy

10 Division of Radiation Oncology, Centro di Riferimento Oncologico di Aviano (CRO) IRCCS, Aviano, Italy

11 Department of Oncology, Radiation Oncology, University of Turin, Turin, Italy

12 Department of Radiation Oncology, ASST Spedali Civili of Brescia, Brescia, Italy

13 Department of Radiation Oncology, S. Croce and Carle Teaching Hospital, Cuneo, Italy and neck cancers amid the COVID-19 pandemic. Adv. Radiat. Oncol. 2020. https://doi.org/10.1016/j.adro.2020.05.010.

33. Wei W, Zheng D, Lei Y, et al. Radiotherapy workflow and protection procedures during the Coronavirus disease 2019 (COVID-19) outbreak: experience of the hubei cancer hospital in Wuhan, China. Radiother Oncol. 2020;148:203-10. https://doi. org/10.1016/j.radonc.2020.03.029.

34. Imbimbo M, Alfieri S, Botta L, et al. Surveillance of patients with head and neck cancer with an intensive clinical and radiologic follow-up. Otolaryngol Neck Surg. 2019;161(4):635-42. https:// doi.org/10.1177/0194599819860808.

35. IARC-International Agency for Research on Cancer. GLOBOCAN cancer fact sheet 2018. https://gco.iarc.fr/today/data/facts heets/cancers/39-All-cancers-fact-sheet.pdf. Accessed 22 June 2020 .

Publisher's Note Springer Nature remains neutral with regard to jurisdictional claims in published maps and institutional affiliations.
14 Dipartimento Diagnostica Per Immagini, Radioterapia Oncologica, UOC Di Radioterapia Oncologica, Fondazione Policlinico Universitario A. Gemelli IRCCS, Rome, Italy

15 National Center of Oncological Hadrontherapy (Fondazione CNAO), Pavia, Italy

16 Department of Radiation Oncology, Azienda Ospedaliera Universitaria Pisana, University of Pisa, Pisa, Italy

17 Radiation Oncology Unit S. Chiara University Hospital of Pisa Via Roma, Pisa, Italy

18 Health Science Department (DISSAL), University of Genova, Genova, Italy

19 Department of Radiotherapy, San Raffaele Scientific Institute, Milan, Italy

20 Department of Radiotherapy, Università Vita Salute S. Raffaele, Milan, Italy

21 Department of Radiation Oncology, IRCCS Regina Elena National Cancer Institute, Rome, Italy

22 Radiation Oncology Division, Oncology and Specialty Medicine Department, San Camillo-Forlanini Hospital, Rome, Italy

23 AIRO (Italian Association of Radiotherapy and Clinical Oncology), Milan, Italy

24 Radiotherapy Unit, Ospedale V Fazzi, Lecce, Italy 